\title{
Role of IFITM3 in polyl: C-induced neuronal impairments
}

\author{
Norimichi Itoh ${ }^{1}$, Taku Nagai ${ }^{1}$, Daisuke Ibi ${ }^{2}$, Akira Nakajima ${ }^{3}$, Toshitaka Nabeshima ${ }^{4}$, \\ Kiyofumi Yamada ${ }^{1}$
}

\begin{abstract}
${ }^{\prime}$ Neuropsychopharmacology and Hospital Pharmacy, Nagoya University Graduate School of Medicine, Japan, ${ }^{2}$ Chemical Pharmacology, Faculty of Pharmaceutical Science, Meijo University, Japan, ${ }^{3}$ Applied Biology and Food Sciences, Faculty of Agriculture and Life Science, Hirosaki University, Japan, ${ }^{4}$ Advanced Diagnostic System Research Laboratory, Fujita Health University Graduate School of Health Science, Japan
\end{abstract}

Epidemiological evidences suggest that perinatal virus infection increases the risk for several psychiatric disorders. We have reported that treatment of polyriboioinic-polyribocytidylic acid (polyI:C), a synthetic double strand RNA which induces natural immune reaction, in neonatal mice lead to schizophrenia-like behavioral abnormality in adulthood. We have found interferon-induced transmembrane protein 3 (IFITM3) in astrocytes as the key molecule in polyI:C-induced behavioral alteration. However, the molecular mechanism underlying roles of IFITM3 in CNS remains unknown. To understand how IFITM3 causes developmental abnormalities in CNS, we screened IFITM3-iteracting proteins and identified Rab GDP dissociation inhibitor (RabGDI) as a novel IFITM3-interactig protein. Interaction of IFITM3 with RabGDI was confirmed by immunoprecipitation and GST pull down assay in HEK293 cells. IFITM3 was partially colocalized with RabGDI in cultured astrocyte. Expression of IFITM3 increased size of EEA1-positive endosome in COS7 cell. GST pull down assay revealed that IFITM3 increased active Rab5. Treatment of condition medium from dominant negative Rab5 expressing astrocyte partially rescued the polyI:C-induced inhibition of neurite elongation. These results suggest that IFITM3 mediates polyI:C-induced neurite impairment by modulating Rab5 activity. 